\title{
Exponential robust stability of T-S fuzzy stochastic $p$-Laplace PDEs under zero-boundary condition
}

Zhilin $\mathrm{Pu}^{1,2^{*}}$ and Ruofeng $\mathrm{RaO}^{2}$

"Correspondence:

puzhilin908@sina.com

${ }^{1}$ College of Mathematics and

Software Science, Sichuan Normal

University, Chengdu, Sichuan

610066, P.R. China

${ }^{2}$ Institution of Mathematics, Yibin

University, Yibin, Sichuan 644007,

P.R. China

\begin{abstract}
In this paper, the stability of a class of time-delay Takagi-Sugeno (T-S) fuzzy Markovian jumping partial differential equations (PDEs) with $p$-Laplace diffusion are investigated, and several criteria for asymptotical stability and robust exponential stability are obtained. Different from all the previous related literature, the authors use the contraction mapping theory to obtain in this paper the existence of other solutions for PDEs with $p$-Laplace besides the trivial solution. In fact, if there is only the trivial solution for the PDEs, the stability criteria about the trivial solution would become meaningless. Moreover, infinitely many solutions for these PDEs of all the previous related literature can be obtained by employing the methods of this paper. In a word, the works of all the related literature were found more meaningful owing to the methods and results of this paper.
\end{abstract}

\section{Introduction}

In this paper, we are to study the stability of a class of delayed Takagi-Sugeno (T-S) fuzzy Markovian jumping $p$-Laplace partial differential equations (PDEs) which owns a wide range of physics and engineering backgrounds (see, e.g., [1-3]). Below, we shall introduce this Markovian jumping fuzzy mathematical model.

Given a complete probability space $(\boldsymbol{\Omega}, \mathcal{F}, \mathbb{P})$ with a natural filtration $\left\{\mathcal{F}_{t}\right\}_{t \geq 0}$, where $\boldsymbol{\Omega}$ is a sample space, $\mathcal{F}$ is $\sigma$-algebra of a subset of the sample space, and $\mathbb{P}$ is the probability measure defined on $\mathcal{F}$. Let $S=\{1,2, \ldots, N\}$ and the random form process $\{r(t), t \in[0,+\infty)\}$ be a homogeneous, finite-state Markovian process with right continuous trajectories with generator $\Pi=\left(\pi_{i j}\right)_{N \times N}$ and transition probability from mode $i$ at time $t$ to mode $j$ at time $t+\Delta t, i, j \in S$,

$$
\mathbb{P}(r(t+\delta)=j \mid r(t)=i)= \begin{cases}\pi_{i j} \delta+o(\delta), & j \neq i, \\ 1+\pi_{i j} \delta+o(\delta), & j=i,\end{cases}
$$

where $\pi_{i j} \geq 0$ is transition probability rate from $i$ to $j(j \neq i)$ and $\pi_{i i}=-\sum_{j=1, j \neq i}^{s} \pi_{i j}, \delta>0$ and $\lim _{\delta \rightarrow 0} o(\delta) / \delta=0$.

Let us consider the following delayed Markovian jumping PDEs:

$$
\begin{aligned}
\frac{\partial u(t, x)}{\partial t}= & \nabla \cdot\left(\mathcal{D}(t, x, u) \circ \nabla_{p} u(t, x)\right)-\mathscr{B}(u(t, x))+\mathcal{C}(r(t), t) f(u(t, x)) \\
& +\mathscr{D}(r(t), t) g(u(t-\tau(r(t), t), x)), \quad t \geq 0, x \in \Omega
\end{aligned}
$$

O2013 Pu and Rao; licensee Springer. This is an Open Access article distributed under the terms of the Creative Commons Attribution License (http://creativecommons.org/licenses/by/2.0), which permits unrestricted use, distribution, and reproduction in any medium, provided the original work is properly cited. 
equipped with the initial condition

$$
u(\theta, x)=\phi(\theta, x), \quad(\theta, x) \in[-\tau, 0] \times \Omega
$$

and the zero-boundary condition

$$
\mathfrak{B}\left[u_{i}(t, x)\right]=0, \quad(t, x) \in[-\tau,+\infty) \times \partial \Omega, i=1,2, \ldots, n,
$$

where $p>1$ is a positive scalar, $\Omega \in R^{m}$ is a bounded domain with a smooth boundary $\partial \Omega$ of class $\mathcal{C}^{2}$ by $\Omega, u(t, x)=\left(u_{1}(t, x), u_{2}(t, x), \ldots, u_{n}(t, x)\right)^{T} \in R^{n}$. Below, $u(t, x)$ is always denoted by $u$ for the sake of convenience. $\mathcal{D}(t, x, u) \circ \nabla_{p} u(t, x)$ denotes the Hadamard product of matrix $\mathcal{D}(t, x, u)$ and $\nabla_{p} u$ (see [1] or [3]), and $\mathcal{D}(t, x, v)=\left(\mathcal{D}_{j k}(t, x, u)\right)_{n \times m}$ satisfies $\mathcal{D}_{j k}(t, x, u) \geq 0$ for all $j, k,(t, x, u)$. In mode $r(t)=i \in S=\{1,2, \ldots, N\}$, we denote $\mathcal{C}_{i}(t)=$ $\mathcal{C}(r(t), t)$ and $\mathscr{D}_{i}(t)=\mathscr{D}(r(t), t)$. Denote by $\tau_{i}(t)$ the time delay $\tau(r(t), t)$, which satisfies $0 \leq$ $\tau_{i}(t) \leq \tau$ for any mode $i \in S$. Functions $\mathscr{B}(u)=\left(\mathscr{B}_{1}\left(u_{1}\right), \mathscr{B}_{2}\left(u_{2}\right), \ldots, \mathscr{B}_{n}\left(u_{n}\right)\right)^{T} \in R^{n}, f(u)=$ $\left(f_{1}\left(u_{1}\right), f_{2}\left(u_{2}\right), \ldots, f_{n}\left(u_{n}\right)\right)^{T} \in R^{n}, g(u)=\left(g_{1}\left(u_{1}\right), g_{2}\left(u_{2}\right), \ldots, g_{n}\left(u_{n}\right)\right)^{T} \in R^{n}$. Boundary condition (1.1b) is called the Dirichlet boundary condition if $\mathfrak{B}\left[u_{i}(t, x)\right]=u_{i}(t, x)$, and the Neumann boundary condition if $\mathfrak{B}\left[u_{i}(t, x)\right]=\frac{\partial u_{i}(t, x)}{\partial v}$. Here, $\frac{\partial u_{i}(t, x)}{\partial v}=\left(\frac{\partial u_{i}(t, x)}{\partial x_{1}}, \frac{\partial u_{i}(t, x)}{\partial x_{2}}, \ldots, \frac{\partial u_{i}(t, x)}{\partial x_{m}}\right)^{T}$ denotes the outward normal derivative on $\partial \Omega$.

Obviously, system (1.1) admits the following Markovian jumping Cohen-Grossberg neural networks as its special case.

$$
\begin{aligned}
\frac{\partial u}{\partial t}= & \nabla \cdot\left(\mathcal{D}(t, x, u) \circ \nabla_{p} u\right) \\
& -\widetilde{A}(u)\left[\widetilde{B}(u)-\widetilde{C}_{i}(t) f(u)-\widetilde{D}_{i}(t) g\left(u\left(t-\tau_{i}(t), x\right)\right)\right] .
\end{aligned}
$$

For mode $i \in S$, system (1.1) is simply denoted as

$$
\frac{\partial u}{\partial t}=\nabla \cdot\left(\mathcal{D}(t, x, u) \circ \nabla_{p} u\right)-\mathscr{B}(u)+\mathcal{C}_{i}(t) f(u)+\mathscr{D}_{i}(t) g\left(u\left(t-\tau_{i}(t), x\right)\right) .
$$

The T-S fuzzy mathematical model with time delay is described as follows.

Fuzzy rule $j$ :

IF $\omega_{1}(t)$ is $\mu_{j 1}$ and $\ldots \omega_{s}(t)$ is $\mu_{j s}$ THEN

$$
\frac{\partial u}{\partial t}=\nabla \cdot\left(\mathcal{D}(t, x, u) \circ \nabla_{p} u\right)-\mathscr{B}(u)+\mathcal{C}_{i j}(t) f(u)+\mathscr{D}_{i j}(t) g\left(u\left(t-\tau_{i}(t), x\right)\right),
$$

where $\omega_{k}(t)(k=1,2, \ldots, s)$ is the premise variable, $\mu_{j k}(j=1,2, \ldots, r ; k=1,2, \ldots, s)$ is the fuzzy set that is characterized by a membership function, $r$ is the number of the IF-THEN rules, and $s$ is the number of the premise variables.

For any mode $r(t)=i \in S$, we assume that $\mathcal{C}_{i j}$, $\mathfrak{D}_{i j}$ are real constant matrices of appropriate dimensions, and $\Delta \mathcal{C}_{i j}, \Delta \mathscr{D}_{i j}$ are real-valued matrix functions which stand for timevarying parameter uncertainties, satisfying

$$
\mathcal{C}_{i j}(t)=\mathcal{C}_{i j}+\Delta \mathcal{C}_{i j}(t), \quad \mathcal{D}_{i j}(t)=\mathscr{D}_{i j}+\Delta \mathscr{D}_{i j}(t)
$$


By way of a standard fuzzy inference method, system (1.4) is inferred as follows:

$$
\begin{aligned}
\frac{\partial u}{\partial t}= & \nabla \cdot\left(\mathcal{D}(t, x, u) \circ \nabla_{p} u\right)-\mathscr{B}(u) \\
& +\sum_{j=1}^{r} h_{j}(\omega(t))\left[\mathcal{C}_{i j}(t) f(u)+\mathcal{D}_{i j}(t) g\left(u\left(t-\tau_{i}(t), x\right)\right)\right],
\end{aligned}
$$

where $\omega(t)=\left[\omega_{1}(t), \omega_{2}(t), \ldots, \omega_{s}(t)\right], h_{j}(\omega(t))=\frac{w_{j}(\omega(t))}{\sum_{k=1}^{r} w_{k}(\omega(t))}, w_{j}(\omega(t)): R^{s} \rightarrow[0,1](j=1,2$, $\ldots, r)$ is the membership function of the system with respect to the fuzzy rule $j . h_{j}$ can be regarded as the normalized weight of each IF-THEN rule, satisfying $h_{j}(\omega(t)) \geq 0$ and $\sum_{j=1}^{r} h_{j}(\omega(t))=1$. Motivated by some methods of the above-mentioned literature and recent related studies $[6-15,22-24]$, we are to investigate the stability of T-S fuzzy system (1.6).

\section{Preparation}

In this paper, we always assume the following.

(H1) Let $\mathcal{B}(u)=\left(\mathscr{B}_{1}\left(u_{1}\right), \mathcal{B}_{2}\left(u_{2}\right), \ldots, \mathcal{B}_{n}\left(u_{n}\right)\right)^{T} \in R^{n}$, there exist positive definite diagonal matrices $\mathbb{B}=\operatorname{diag}\left(b_{1}, b_{2}, \ldots, b_{n}\right)$ and $\widetilde{\mathbb{B}}=\operatorname{diag}\left(\widetilde{b}_{1}, \widetilde{b}_{2}, \ldots, \widetilde{b}_{n}\right)$ such that

$$
\widetilde{b}_{j} \geq \sup _{r \in R} \mathcal{B}_{j}^{\prime}(r) \geq \inf _{r \in R} \mathcal{B}_{j}^{\prime}(r) \geq b_{j}, \quad \forall j=1,2, \ldots, n
$$

(H2) There exist constant diagonal matrices $G_{k}=\operatorname{diag}\left(G_{1}^{(k)}, G_{2}^{(k)}, \ldots, G_{n}^{(k)}\right)$, $F_{k}=\operatorname{diag}\left(F_{1}^{(k)}, F_{2}^{(k)}, \ldots, F_{n}^{(k)}\right), k=1,2$, with $\left|F_{j}^{(1)}\right| \leq F_{j}^{(2)},\left|G_{j}^{(1)}\right| \leq G_{j}^{(2)}, j=1,2, \ldots, n$, such that

$$
F_{j}^{(1)} \leq \frac{f_{j}(r)}{r} \leq F_{j}^{(2)}, \quad G_{j}^{(1)} \leq \frac{g_{j}(r)}{r} \leq G_{j}^{(2)}, \quad \forall j=1,2, \ldots, n, \text { and } r \in R
$$

In addition, one can assume that $u=0$ is a trivial solution of PDEs (1.6) provided that $\mathcal{B}(0)=f(0)=g(0)=0$. For any mode $i \in S$, the parameter uncertainties considered here are norm-bounded and of the following forms:

$$
\left(\Delta \mathcal{C}_{i j}(t) \quad \Delta \mathscr{D}_{i j}(t)\right)=E_{i j} \mathfrak{F}(t)\left(H_{i j} \quad M_{i j}\right), \quad \forall i \in S
$$

Here $\mathfrak{F}(t)$ is an unknown matrix function satisfying $\left|\mathfrak{F}^{T}(t) \| \mathfrak{F}(t)\right| \leq I$, and $E_{i j}, G_{i j}, H_{i j}$ are known real constant matrices. Throughout this paper, for a matrix $C=\left(c_{i j}\right)_{n \times n}$, we denote the matrix $|C|=\left(\left|c_{i j}\right|\right)_{n \times n}$. In addition, we denote by $I$ the identity matrix with compatible dimension and denote $\|u\|_{2}^{2}=\int_{\Omega} u^{2}(t, x) d x$.

Definition 2.1 For any given scalar $p>1$, system (1.6) is said to be global stochastic exponential robust stability in the mean square if for every initial condition $\phi \in L_{\mathcal{F}_{0}}^{2}([-\tau, 0] \times$ $\left.\Omega ; R^{n}\right), r(0)=i_{0}$, there exist scalars $\beta>0$ and $\gamma>0$ such that for any solution $u\left(t, x ; \phi, i_{0}\right)$,

$$
\mathbb{E}\left(\left\|u\left(t, x ; \phi, i_{0}\right)\right\|_{2}^{2}\right) \leq \gamma e^{-\beta t}\left[\sup _{-\tau \leq \theta \leq 0} \mathbb{E}\left(\|\phi(\theta, x)\|_{2}^{2}\right)\right], \quad t \geq 0
$$

for all admissible uncertainties satisfying (2.1). 
Note that Definition 2.1 actually provides the definition about the global stochastic exponential robust stability for the trivial solution $u=0$ of PDEs (1.6).

Lemma 2.1 ([4]) Let $\varepsilon>0$ be any given scalar, and let $\mathcal{M}, \mathfrak{E}$ and $\mathcal{K}$ be matrices with appropriate dimensions. If $\mathcal{K}^{T} \mathcal{K} \leq I$, then we have

$$
\mathcal{M K E}+\mathfrak{E}^{T} \mathcal{K}^{T} \mathcal{M}^{T} \leq \varepsilon^{-1} \mathcal{M} \mathcal{M}^{T}+\varepsilon \mathfrak{E}^{T} \mathfrak{E} .
$$

Lemma 2.2 (Schur complement [5]) Given matrices $\mathcal{Q}(t), \mathcal{S}(t)$ and $\mathcal{R}(t)$ with appropriate dimensions, where $\mathcal{Q}(t)=\mathcal{Q}(t)^{T}, \mathcal{R}(t)=\mathcal{R}(t)^{T}$, then

$$
\left(\begin{array}{cc}
\mathcal{Q}(t) & \mathcal{S}(t) \\
\mathcal{S}^{T}(t) & \mathcal{R}(t)
\end{array}\right)>0
$$

if and only if

$$
\mathcal{R}(t)>0, \quad \mathcal{Q}(t)-\mathcal{S}(t) \mathcal{R}^{-1}(t) \mathcal{S}^{T}(t)>0
$$

or

$$
\mathcal{Q}(t)>0, \quad \mathcal{R}(t)-\mathcal{S}^{T}(t) \mathcal{Q}^{-1}(t) \mathcal{S}^{T}(t)>0,
$$

where $\mathcal{Q}(t), \mathcal{S}(t)$ and $\mathcal{R}(t)$ are dependent on $t$.

\section{Main result}

In this section, we assume that the time-varying delays $\tau_{i}(t)$ satisfy $0 \leq \tau_{i}(t) \leq \tau$ with $\dot{\tau}_{i}(t)+$ $\sum_{l \in S} \pi_{i l} \tau_{l}(t) \leq a_{0}<1$ for any mode $i \in S$.

Theorem 3.1 Let $p>1$. Assume that there exist a positive scalar $\alpha<1$ and a sequence of positive scalars $c_{j k}^{i l}$ and $d_{j k}^{i l}$ with $\left|c_{j k}^{i l}(t)\right| \leq c_{j k}^{i l}$ and $\left|d_{j k}^{i l}(t)\right| \leq d_{j k}^{i l}$ for all $i, k, t \geq 0$ such that

$$
\max _{j}\left(\frac{\widetilde{b}_{j}-b_{j}}{b_{j}}\right)+\left(\max _{k}\left(\left|c_{j k}^{i l}\right| F_{k}^{(2)}\right)+\max _{k}\left(\left|d_{j k}^{i l}\right| G_{k}^{(2)}\right)\right) \sum_{j=1}^{n} \frac{r}{b_{j}} \leq \alpha<1 .
$$

If, in addition, there exist a positive scalar $\beta>0$ and positive definite diagonal matrices $P_{i}(i \in S), L_{1}, L_{2}$ and $Q$ such that the following LMI conditions hold:

$$
\left(\begin{array}{cc}
\Omega_{i 1} & \Omega_{i 2} \\
* & \Omega_{i 3}
\end{array}\right)<0, \quad \forall i \in S
$$

where $\mathcal{C}_{i l}(t)=\left(c_{j k}^{i l}(t)\right)_{n \times n}, \mathscr{D}_{i l}(t)=\left(d_{j k}^{i l}(t)\right)_{n \times n}$,

$$
\begin{aligned}
& \Omega_{i 1}=\left(\begin{array}{cccccc}
\mathcal{A}_{i 1} & 0 & \left(F_{1}+F_{2}\right) L_{1}+P_{i} \sum_{j=1}^{r}\left|\mathcal{C}_{i j}\right| & P_{i} \sum_{j=1}^{r}\left|\mathcal{D}_{i j}\right| \\
* & \mathcal{A}_{i 2} & 0 & & \left(G_{1}+G_{2}\right) L_{2} \\
* & * & -2 L_{1} & & 0 \\
* & * & & * & & -2 L_{2}
\end{array}\right), \\
& \Omega_{i 2}=\left(\begin{array}{llllllll}
\mathcal{M}_{1} & \mathfrak{E}_{1}^{T} & \mathcal{M}_{2} & \mathfrak{E}_{2}^{T} & \ldots & \ldots & \mathcal{M}_{r} & \mathfrak{E}_{r}^{T}
\end{array}\right),
\end{aligned}
$$




$$
\begin{aligned}
& \Omega_{i 3}=\left(\begin{array}{cccccccc}
-I_{1} & 0 & 0 & 0 & 0 & 0 & 0 & 0 \\
* & -I_{2} & 0 & 0 & 0 & 0 & 0 & 0 \\
* & * & -I_{3} & 0 & 0 & 0 & 0 & 0 \\
* & * & * & -I_{4} & 0 & 0 & 0 & 0 \\
* & * & * & * & \ddots & 0 & 0 & 0 \\
* & * & * & * & \ldots & * & -I_{2 r-1} & 0 \\
* & * & * & * & * & \ldots & * & -I_{2 r}
\end{array}\right), \\
& \mathcal{A}_{i 1}=-2 P_{i} \mathbb{B}+\sum_{l \in S} \pi_{i l} P_{l}+\beta P_{i}+Q-2 F_{1} L_{1} F_{2}, \quad \mathcal{A}_{i 2}=-\left(1-a_{0}\right) e^{-\tau \beta} Q-2 G_{1} L_{2} G_{2}, \\
& I_{j}=I, \quad \forall j=1,2, \ldots, 2 r, \\
& \mathcal{M}_{j}=\left(\begin{array}{c}
P_{i}\left|E_{i j}\right| \\
0 \\
0 \\
0
\end{array}\right), \quad \mathfrak{E}_{j}=\left(\begin{array}{c}
0 \\
0 \\
\left|H_{i j}^{T}\right| \\
\left|M_{i j}^{T}\right|
\end{array}\right)^{T},
\end{aligned}
$$

then system (1.6) is global stochastic exponential robust stability in the mean square.

Proof The whole proof may be divided into two big steps.

Step 1. Firstly show the existence of solutions for system (1.6) though it owns a trivial solution obviously. We may consider the existence of a class of solutions that $u(t, x)=$ $u(t)$ for all $x \in \Omega$, and $\mathfrak{B}[u(t, x)]=0$ for all $(t, x) \in[-\tau,+\infty) \times \partial \Omega$. For any given initial condition $\phi(\theta)$, we show that there exists at least the above-mentioned solution for PDEs (1.6), satisfying the initial condition $u(\theta)=\phi(\theta)$ for all $\theta \in[-\tau, 0]$. So, in this section, we only need to consider the existence of solutions for the following system:

$$
\left\{\begin{array}{l}
\frac{d u(t)}{d t}=-\mathscr{B}(u(t))+\sum_{j=1}^{r} h_{j}(\omega(t))\left[\mathcal{C}_{i j}(t) f(u(t))+\mathscr{D}_{i j}(t) g\left(u\left(t-\tau_{i}(t)\right)\right)\right], \quad t \geq 0, i \in S, \\
u(\theta)=\phi(\theta), \quad-\tau \leq \theta \leq 0
\end{array}\right.
$$

or equivalently,

$$
\left\{\begin{aligned}
\frac{d u_{j}(t)}{d t}= & -\mathcal{B}_{j}\left(u_{j}(t)\right)+\sum_{l=1}^{r} h_{l}(\omega(t))\left[\sum_{k=1}^{n} c_{j k}^{i l}(t) f_{k}\left(u_{k}(t)\right)\right. \\
& \left.+\sum_{k=1}^{n} d_{j k}^{i l}(t) g_{k}\left(u_{k}\left(t-\tau_{i}(t)\right)\right)\right], \quad t \geq 0, i \in S, \forall j \\
u_{j}(\theta)= & \phi_{j}(\theta), \quad-\tau \leq \theta \leq 0, \forall j
\end{aligned}\right.
$$

where $j=1,2, \ldots, n$.

To apply the fixed point theory, we need to define the complete metric space as follows.

Let $\Theta=\Theta_{1} \times \Theta_{2} \times \cdots \times \Theta_{n}$, and let $\Theta_{j}(j=1,2, \ldots, n)$ be the space consisting of functions $q_{j}(t):[-\tau, \infty) \rightarrow R$ satisfying

(a) $q_{j}(t)$ is continuous on $t \in[0, \infty)$;

(b) $q_{j}(\theta)=\phi_{j}(\theta),-\tau \leq \theta \leq 0$. 
In fact, it is not difficult to verify that the above space $\Theta$ is a complete space provided that it is equipped with the following metric:

$$
\operatorname{dist}(\hat{q}, \breve{q})=\sum_{j=1}^{n}\left(\sup _{t \geq-\tau}\left|\hat{q}_{j}(t)-\breve{q}_{j}(t)\right|\right),
$$

where $\hat{q}=\hat{q}(t)=\left(\hat{q}_{1}(t), \hat{q}_{2}(t), \ldots, \hat{q}_{n}(t)\right)^{T} \in \Theta$ and $\breve{q}=\breve{q}(t)=\left(\breve{q}_{1}(t), \breve{q}_{2}(t), \ldots, \breve{q}_{n}(t)\right)^{T} \in \Theta$.

Define the operator $\Phi$ acting on $\Theta$ for $u(t)=\left(u_{1}(t), \ldots, u_{j}(t), \ldots, u_{n}(t)\right)^{T} \in \Theta$ such that $\Phi(u)(t)=\left(\Phi\left(u_{1}\right)(t), \ldots, \Phi\left(u_{j}\right)(t), \ldots, \Phi\left(u_{n}\right)(t)\right)$, where $\Phi\left(u_{j}\right)(t):[-\tau, \infty) \rightarrow R$ satisfies

$$
\begin{aligned}
\Phi\left(u_{j}\right)(t)= & e^{-b_{j} t} \phi_{j}(0)+e^{-b_{j} t} \int_{0}^{t} e^{b_{j} s}\left[-\left(\mathcal{B}_{j}\left(u_{j}(s)\right)-b_{j} u_{j}(s)\right)\right. \\
& \left.+\sum_{l=1}^{r} h_{l}(\omega(s))\left(\sum_{k=1}^{n} c_{j k}^{i l}(s) f_{k}\left(u_{k}(s)\right)+\sum_{k=1}^{n} d_{j k}^{i l}(s) g_{k}\left(u_{k}\left(s-\tau_{i}(s)\right)\right)\right)\right] d s,
\end{aligned}
$$

$$
t \geq 0, i \in S
$$

and $\Phi\left(u_{j}\right)(\theta)=\phi_{j}(\theta),-\tau \leq \theta \leq 0$ for each $j=1,2, \ldots, n$.

In view of the fact that $\Phi\left(u_{j}\right)(0)=\phi_{j}(0)$ for each $j$, it is not difficult to verify that the fixed point of $\Phi$ is the solution of system (3.3), and the solution of (3.3) is the fixed point of $\Phi$. So we only need to prove that $\Phi$ has the corresponding fixed point on $\Theta$ for any given initial condition $\phi(\theta)$.

Next, we claim that $\Phi\left(\Theta_{j}\right) \subset \Theta_{j}$ for each $j=1,2, \ldots, n$.

Indeed, for any given $u_{j}(t) \in \Theta_{j}$, it follows from (3.4) and the assumptions on $f_{j}, g_{j}$ that $\Phi\left(u_{j}\right)(t)$ is continuous on $t \in[0, \infty)$, and hence condition (a) is satisfied. In addition, $\Phi\left(u_{j}\right)(\theta)=\phi_{j}(\theta),-\tau \leq \theta \leq 0$, and then condition (b) holds. Thereby, $\Phi\left(\Theta_{j}\right) \subset \Theta_{j}$ for all $j$.

Finally, we claim that $\Phi$ is a contraction mapping on $\Theta$.

Indeed, for $u(t)=\left(u_{1}(t), \ldots, u_{j}(t), \ldots, u_{n}(t)\right)^{T} \in \Theta, v(t)=\left(v_{1}(t), \ldots, v_{j}(t), \ldots, v_{n}(t)\right)^{T} \in \Theta$, we have

$$
\left|\Phi\left(u_{j}\right)(t)-\Phi\left(v_{j}\right)(t)\right| \leq J_{j 1}+J_{j 2}+J_{j 3}
$$

where

$$
\begin{aligned}
& J_{j 1}=e^{-b_{j} t} \int_{0}^{t} e^{b_{j} s}\left[\left|\left(\mathcal{B}_{j}\left(u_{j}(s)\right)-b_{j} u_{j}(s)\right)-\left(\mathcal{B}_{j}\left(v_{j}(s)\right)-b_{j} v_{j}(s)\right)\right|\right] d s, \\
& J_{j 2}=e^{-b_{j} t} \int_{0}^{t} e^{b_{j} s} \sum_{l=1}^{r}\left|h_{l}(\omega(s))\right|\left(\sum_{k=1}^{n}\left|c_{j k}^{i l}(s)\right|\left|f_{k}\left(u_{k}(s)\right)-f_{k}\left(v_{k}(s)\right)\right|\right) d s, \\
& J_{j 3}=e^{-b_{j} t} \int_{0}^{t} e^{b_{j} s} \sum_{l=1}^{r}\left|h_{l}(\omega(s))\right|\left(\sum_{k=1}^{n}\left|d_{j k}^{i l}(s)\right|\left|g_{k}\left(u_{k}\left(s-\tau_{i}(s)\right)\right)-g_{k}\left(v_{k}\left(s-\tau_{i}(s)\right)\right)\right|\right) d s .
\end{aligned}
$$

We can get, by the differential mean value theorem,

$$
J_{j 1} \leq\left(\widetilde{b}_{j}-b_{j}\right) \sup _{t \geq 0}\left|u_{j}(t)-v_{j}(t)\right| e^{-b_{j} t} \int_{0}^{t} e^{b_{j} s} d s \leq \frac{\widetilde{b}_{j}-b_{j}}{b_{j}} \sup _{t \geq-\tau}\left|u_{j}(t)-v_{j}(t)\right|,
$$


and obtain, by the assumptions on $f, g$,

$$
J_{j 2} \leq \frac{r}{b_{j}} \sum_{k=1}^{n}\left|c_{j k}^{i l}\right| F_{k}^{(2)} \sup _{t \geq-\tau}\left|u_{k}(t)-v_{k}(t)\right| \leq \frac{r}{b_{j}} \max _{k}\left(\left|c_{j k}^{i l}\right| F_{k}^{(2)}\right) \operatorname{dist}(u, v)
$$

and

$$
\begin{aligned}
J_{j 3} & \leq r \sum_{k=1}^{n}\left|d_{j k}^{i l}\right| G_{k}^{(2)} \sup _{t \geq 0}\left|u_{k}\left(t-\tau_{i}(t)\right)-v_{k}\left(t-\tau_{i}(t)\right)\right| e^{-b_{j} t} \int_{0}^{t} e^{b_{j} s} d s \\
& \leq \frac{r}{b_{j}} \max _{k}\left(\left|d_{j k}^{i l}\right| G_{k}^{(2)}\right) \operatorname{dist}(u, v) .
\end{aligned}
$$

Then we have

$$
\begin{aligned}
\sup _{t \geq-\tau}\left|\Phi\left(u_{j}\right)(t)-\Phi\left(v_{j}\right)(t)\right| \leq & \frac{\widetilde{b}_{j}-b_{j}}{b_{j}} \sup _{t \geq-\tau}\left|u_{j}(t)-v_{j}(t)\right|+\frac{r}{b_{j}} \max _{k}\left(\left|c_{j k}^{i l}\right| F_{k}^{(2)}\right) \operatorname{dist}(u, v) \\
& +\frac{r}{b_{j}} \max _{k}\left(\left|d_{j k}^{i l}\right| G_{k}^{(2)}\right) \operatorname{dist}(u, v) .
\end{aligned}
$$

Therefore,

$$
\begin{aligned}
\operatorname{dist} & (\Phi(u), \Phi(v)) \\
= & \sum_{j=1}^{n} \sup _{t \geq-\tau}\left|\Phi\left(u_{j}\right)(t)-\Phi\left(v_{j}\right)(t)\right| \\
\leq & {\left[\max _{j}\left(\frac{\widetilde{b}_{j}-b_{j}}{b_{j}}\right)+\left(\max _{k}\left(\left|c_{j k}^{i l}\right| F_{k}^{(2)}\right)+\max _{k}\left(\left|d_{j k}^{i l}\right| G_{k}^{(2)}\right)\right) \sum_{j=1}^{n} \frac{r}{b_{j}}\right] \operatorname{dist}(u, v) } \\
\leq & \alpha \operatorname{dist}(u, v),
\end{aligned}
$$

which implies that $\Phi$ is a contraction mapping on $\Theta$. And then the contraction mapping theory yields that $\Phi$ has the fixed point $u(t)$ on $\Theta$, which means $u(t)$ is the solution to system (3.3) for a given initial condition $\phi(\theta)$.

Remark 3.1 If the number of the IF-THEN rules $r=1$, then (1.6) is just (1.1). Hence, we have also shown the existence of solutions for (1.1).

Remark 3.2 From the arbitrariness of initial condition $\phi(\theta)$, we know that system (1.6) may in all probability own infinitely many solutions under zero-boundary condition (1.1b). Below, we shall show that all the above-mentioned solutions converge to the trivial solution $u=0$ as $t \rightarrow \infty$ for an arbitrary initial condition $\phi(\theta)$. In fact, we shall prove that the trivial solution of PDEs (1.6) is globally exponentially stable. However, in much previous related literature (e.g., [3]), the existence of solutions for PDEs is not discussed. Naturally, people want to ask whether the system owns the other solutions besides the trivial solution. Now we provide a sufficient condition for the existence. Compared with [3], it is a major advance. In addition, we provide the first method, by which we can also give similar sufficient conditions for the existence of the PDEs in the previous related literature, including [3]. 
Step 2. We prove that system (1.6) is global stochastic exponential robust stability in the mean square.

Consider the Lyapunov-Krasovskii functional:

$$
V(t, i)=V_{1 i}+V_{2 i}, \quad \forall i \in S
$$

where

$$
\begin{aligned}
& V_{1 i}=e^{\beta t} \int_{\Omega} u^{T}(t, x) P_{i} u(t, x) d x, \\
& V_{2 i}=e^{\beta t} \int_{\Omega} \int_{-\tau_{i}(t)}^{0} e^{\beta \theta} u^{T}(t+\theta, x) Q u(t+\theta, x) d \theta d x .
\end{aligned}
$$

$\mathcal{L}$ is the weak infinitesimal operator such that $\mathcal{L} V(t, u(t, x), i)=\mathcal{L} V_{1 i}+\mathcal{L} V_{2 i}$ for any given $i \in S$. Then we have

$$
\begin{aligned}
\mathcal{L} V_{1 i}= & e^{\beta t}\left\{2 \int_{\Omega} u^{T} P_{i}\left(\nabla \cdot\left(\mathcal{D}(t, x, u) \circ \nabla_{p} u\right)\right) d x-2 \int_{\Omega} u^{T} P_{i} \mathcal{B}(u(t, x)) d x\right. \\
& +2 \int_{\Omega} \sum_{j=1}^{r} h_{j}(\omega(t))\left[u^{T} P_{i} \mathcal{C}_{i j}(t) f(u)+u^{T} P_{i} \mathcal{D}_{i j}(t) g\left(u\left(t-\tau_{i}(t), x\right)\right)\right] d x \\
& \left.+\int_{\Omega} u^{T}\left(\sum_{l \in S} \pi_{i l} P_{l}\right) u d x\right\}+\beta e^{\beta t} \int_{\Omega} u^{T} P_{i} u d x,
\end{aligned}
$$

where $u=u(t, x)$ is a solution for PDEs (1.6).

$$
\begin{aligned}
\mathcal{L} V_{2 i}= & e^{\beta t} \int_{\Omega} u^{T} Q u d x \\
& -\left(1-\dot{\tau}_{i}(t)-\sum_{l \in S} \pi_{i l} \tau_{l}(t)\right) e^{\beta\left(t-\tau_{i}(t)\right)} \int_{\Omega} u^{T}\left(t-\tau_{i}(t), x\right) Q u\left(t-\tau_{i}(t), x\right) d x \\
\leq & e^{\beta t}\left[\int_{\Omega} u^{T} Q u d x-\left(1-a_{0}\right) e^{-\tau \beta} \int_{\Omega} u^{T}\left(t-\tau_{i}(t), x\right) Q u\left(t-\tau_{i}(t), x\right) d x\right] \\
= & e^{\beta t}\left[\int_{\Omega}\left|u^{T}\right| Q|u| d x-\left(1-a_{0}\right) e^{-\tau \beta} \int_{\Omega}\left|u^{T}\left(t-\tau_{i}(t), x\right)\right| Q\left|u\left(t-\tau_{i}(t), x\right)\right| d x\right] .
\end{aligned}
$$

It follows immediately by [3, Lemma 6$]$ that

$$
2 \int_{\Omega} u^{T} P_{i}\left(\nabla \cdot\left(\mathcal{D}(t, x, u) \circ \nabla_{p} u\right)\right) d x \leq 0 .
$$

Condition (H1) derives that

$$
\int_{\Omega} u^{T} P_{i} \mathcal{B}(u(t, x)) d x \geq \int_{\Omega} u^{T} P_{i} \mathbb{B} u d x=\int_{\Omega}\left|u^{T}\right| P_{i} \mathbb{B}|u| d x .
$$

From (H2), we have

$$
2\left|f^{T}(u)\right| L_{1}|f(u)|-2\left|u^{T}\right|\left(F_{1}+F_{2}\right) L_{1}|f(u)|+2\left|u^{T}\right| F_{1} L_{1} F_{2}|u| \leq 0,
$$




$$
\begin{aligned}
& 2\left|g^{T}\left(u\left(t-\tau_{i}(t), x\right)\right)\right| L_{2}\left|g\left(u\left(t-\tau_{i}(t), x\right)\right)\right|+2\left|u^{T}\left(t-\tau_{i}(t), x\right)\right| G_{1} L_{2} G_{2}\left|u\left(t-\tau_{i}(t), x\right)\right| \\
& \quad \leq 2\left|u^{T}\left(t-\tau_{i}(t), x\right)\right|\left(G_{1}+G_{2}\right) L_{2}\left|g\left(u\left(t-\tau_{i}(t), x\right)\right)\right| .
\end{aligned}
$$

Combining the above analysis results in

$$
\mathcal{L} V(t, v(t, x), i) \leq e^{\beta t} \int_{\Omega} \zeta^{T}(t, x) \mathfrak{A}_{i} \zeta(t, x) d x
$$

where

$$
\mathfrak{A}_{i}=\left(\begin{array}{cccc}
\mathcal{A}_{i 1} & 0 & \left(F_{1}+F_{2}\right) L_{1}+P_{i} \sum_{j=1}^{r} h_{j}(\omega(t)) \mathcal{C}_{i j}(t) & P_{i} \sum_{j=1}^{r} h_{j}(\omega(t)) D_{i j}(t) \\
* & \mathcal{A}_{i 2} & 0 & \left(G_{1}+G_{2}\right) L_{2} \\
* & * & -2 L_{1} & 0 \\
* & * & * & -2 L_{2}
\end{array}\right)
$$

and $\zeta(t, x)=\left(\left|u^{T}(t, x)\right|,\left|u^{T}(t-\tau(t), x)\right|,\left|f^{T}(u(t, x))\right|,\left|g^{T}\left(u\left(t-\tau_{i}(t), x\right)\right)\right|\right)^{T}$.

In addition, we have

$$
\begin{aligned}
& 2 \int_{\Omega} u^{T} P_{i} \sum_{j=1}^{r} h_{j}(\omega(t)) \mathcal{C}_{i j}(t) f(u(t, x)) d x \\
& \quad \leq 2 \int_{\Omega}\left[\left|u^{T}\right| P_{i} \sum_{j=1}^{r}\left(\left|\mathcal{C}_{i j}\right|+\left|\Delta \mathcal{C}_{i j}\right|\right)|f(u(t, x))|\right] d x \\
& \quad \leq 2 \int_{\Omega}\left[\left|u^{T}\right| P_{i} \sum_{j=1}^{r}\left(\left|\mathcal{C}_{i j}\right|+\left|E_{i j}\right||\mathfrak{F}(t)|\left|H_{i j}\right|\right)|f(u(t, x))|\right] d x .
\end{aligned}
$$

Similarly,

$$
\begin{aligned}
& 2 \int_{\Omega} u^{T} P_{i} \sum_{j=1}^{r} h_{j}(\omega(t)) D_{i j}(t) g\left(u\left(t-\tau_{i}(t), x\right)\right) d x \\
& \quad \leq 2 \int_{\Omega}\left[\left|u^{T}\right| P_{i} \sum_{j=1}^{r}\left(\left|D_{i j}\right|+\left|E_{i j}\right||\mathfrak{F}(t)|\left|M_{i j}\right|\right)\left|g\left(u\left(t-\tau_{i}(t), x\right)\right)\right|\right] d x .
\end{aligned}
$$

Further, we can apply the Schur complement to (3.2) and derive $\mathfrak{A}_{i}<0$. Hence, $\mathcal{L} V(t, i) \leq 0$. Define

$$
\mathcal{V}(t, i)=\int_{\Omega} u^{T}(t, x) P_{i} u(t, x) d x+\int_{\Omega} \int_{-\tau_{i}(t)}^{0} e^{\beta \theta} u^{T}(t+\theta, x) Q u(t+\theta, x) d \theta d x
$$

Then we get $V(t, i)=e^{\beta t} \mathcal{V}(t, i)$, satisfying $\mathcal{L}\left(e^{\beta t} \mathcal{V}(t, i)\right)=\mathcal{L} V(t, i) \leq 0$.

Further, by applying the Dynkin formula, we can derive that for any $i \in S$,

$$
e^{\beta t} \mathbb{E} \mathcal{V}(t)-\mathbb{E} \mathcal{V}(0)=\mathbb{E} \int_{0}^{t} \mathcal{L}\left(e^{\beta s} \mathcal{V}(s)\right) d s \leq 0 \quad \text { for all } t \geq 0
$$


Now, for any $\phi(\theta, x) \in L_{\mathcal{F}_{0}}^{2}\left([-\tau, 0] \times \Omega ; R^{n}\right)$ and any system mode $i \in S$, the solution $u\left(t, x, \phi, i_{0}\right)$ of system (1.6) with the initial value $\phi$ satisfies

$$
\begin{aligned}
\min _{i \in S} & \left\{\underline{\alpha}_{i}\right\} e^{\beta t} \mathbb{E}\left(\left\|u\left(t, x, \phi, i_{0}\right)\right\|_{2}^{2}\right) \\
& \leq \underline{\alpha}_{i} e^{\beta t} \mathbb{E}\left(\left\|u\left(t, x, \phi, i_{0}\right)\right\|_{2}^{2}\right) \\
& \leq e^{\beta t} \mathbb{E} \mathcal{V}(t, u(t, x), i) \leq \mathbb{E} \mathcal{V}(0, u(0, x), i) \\
& \leq \mathbb{E}\left(\bar{\alpha}_{i}\|\phi(0)\|_{2}^{2}\right)+\mathbb{E}\left(\int_{-\tau}^{0} \int_{\Omega} e^{\beta \theta}\left[\phi^{T}(\theta, x) Q \phi(\theta, x)\right] d x d \theta\right) \\
& \leq\left(\max _{i \in S}\left\{\bar{\alpha}_{i}\right\}+\lambda_{\max } Q\right) \sup _{-\tau \leq \theta \leq 0} \mathbb{E}\left(\|\phi(\theta)\|_{2}^{2}\right), \quad t \geq 0,
\end{aligned}
$$

or

$$
\mathbb{E}\left(\left\|v\left(t, x ; \phi, i_{0}\right)\right\|_{2}^{2}\right) \leq \gamma e^{-\beta t} \sup _{-\tau \leq \theta \leq 0} \mathbb{E}\left(\|\phi(\theta, x)\|_{2}^{2}\right), \quad t \geq 0,
$$

where positive scalars $\underline{\alpha}_{i}, \bar{\alpha}_{i}$ satisfy $\underline{\alpha}_{i} I \leq P_{i}$ and $\bar{\alpha} I \geq P_{i}$ for any mode $i \in S$, scalars $\gamma=$ $\frac{1}{\min _{i \in S}\left\{\underline{\alpha}_{i}\right\}}\left(\max _{i \in S}\left\{\bar{\alpha}_{i}\right\}+\lambda_{\max } Q\right)>0, \beta>0$. Therefore, we can see by (3.13) and Definition 2.1 that system (1.6) is global stochastic exponential robust stability in the mean square.

Remark 3.3 It is the first time to obtain the robust exponential stability criterion for T$S$ fuzzy PDEs (1.6). Theorem 3.1 admits more effectiveness and less conservatism due to the large allowable variation range of time-delay, which will be illustrated by a numerical example (below).

Remark 3.4 System (1.6) encompasses a wide range of physics and engineering backgrounds so that we can apply our Theorem 3.1 to the case of Cohen-Grossberg neural networks. We consider the following T-S fuzzy Markovian jumping CGNNs with $p$-Laplace diffusion:

$$
\begin{aligned}
\frac{\partial u}{\partial t}= & \nabla \cdot\left(\mathcal{D}(t, x, u) \circ \nabla_{p} u\right) \\
& -A(u)\left\{\mathcal{B}(u)-\sum_{j=1}^{r} h_{j}(\omega(t))\left[\mathcal{C}_{i j}(t) f(u)+\mathscr{D}_{i j}(t) g\left(u\left(t-\tau_{i}(t), x\right)\right)\right]\right\},
\end{aligned}
$$

where $A(u)=\operatorname{diag}\left(a_{1}\left(u_{1}(t, x)\right), a_{2}\left(u_{2}(t, x)\right), \ldots, a_{n}\left(u_{n}(t, x)\right)\right)$, satisfying

$$
0<\underline{a}_{j} \leq a_{j}(r) \leq \bar{a}_{j}, \quad j=1,2, \ldots, n .
$$

Denote $\underline{A}=\operatorname{diag}\left(\underline{a}_{1}, \underline{a}_{2}, \ldots, \underline{a}_{n}\right)$ and $\bar{A}=\operatorname{diag}\left(\bar{a}_{1}, \bar{a}_{2}, \ldots, \bar{a}_{n}\right)$.

Corollary 3.2 Assume $p>1$. CGNNs (3.14) is global exponential robust stability in the mean square if there exist a positive scalar $\beta>0$ and positive definite diagonal matrices $P_{i}$ $(i \in S), L_{1}, L_{2}$ and $Q$ such that the following LMI conditions hold:

$$
\left(\begin{array}{cc}
\widetilde{\Omega}_{i 1} & \widetilde{\Omega}_{i 2} \\
* & \Omega_{i 3}
\end{array}\right)<0, \quad \forall i \in S,
$$


where

$$
\begin{aligned}
& \widetilde{\Omega}_{i 1}=\left(\begin{array}{cccc}
\widetilde{\mathcal{A}}_{i 1} & 0 & \left(F_{1}+F_{2}\right) L_{1}+P_{i} \bar{A} \sum_{j=1}^{r}\left|\mathcal{C}_{i j}\right| & P_{i} \bar{A} \sum_{j=1}^{r}\left|D_{i j}\right| \\
* & \mathcal{A}_{i 2} & 0 & \left(G_{1}+G_{2}\right) L_{2} \\
* & * & -2 L_{1} & 0 \\
* & * & * & -2 L_{2}
\end{array}\right), \\
& \widetilde{\Omega}_{i 2}=\left(\begin{array}{llllllll}
\widetilde{\mathcal{M}}_{1} & \mathfrak{E}_{1}^{T} & \widetilde{\mathcal{M}}_{2} & \mathfrak{E}_{2}^{T} & \ldots & \ldots & \widetilde{\mathcal{M}}_{r} & \mathfrak{E}_{r}^{T}
\end{array}\right), \\
& \widetilde{\mathcal{M}}_{j}=\left(\begin{array}{c}
P_{i} \bar{A}\left|E_{i j}\right| \\
0 \\
0 \\
0
\end{array}\right), \quad \mathfrak{E}_{j}=\left(\begin{array}{c}
0 \\
0 \\
\left|H_{i j}^{T}\right| \\
\left|M_{i j}^{T}\right|
\end{array}\right)^{T} \text {, } \\
& \widetilde{\mathcal{A}}_{i 1}=-2 P_{i} \underline{A} \mathbb{B}+\sum_{l \in S} \pi_{i l} P_{l}+\beta P_{i}+Q-2 F_{1} L_{1} F_{2} .
\end{aligned}
$$

\section{A numerical example}

Example 4.1 Consider the T-S fuzzy $p$-Laplace PDEs with Markovian jumping parameters as follows.

Fuzzy rule 1:

IF $\omega_{1}(t)$ is $\mu_{11}$ and $\ldots \omega_{s}(t)$ is $\mu_{1 s}$, THEN

$$
\begin{aligned}
\frac{\partial u}{\partial t}= & \nabla \cdot\left(\mathcal{D}(t, x, u) \circ \nabla_{p} u\right)-\mathscr{B}(u)+\mathcal{C}_{i 1}(t) f(u) \\
& +\mathscr{D}_{i 1}(t) g\left(u\left(t-\tau_{i}(t), x\right)\right), \quad i \in S .
\end{aligned}
$$

Fuzzy rule 2:

IF $\omega_{2}(t)$ is $\mu_{21}$ and $\ldots \omega_{s}(t)$ is $\mu_{2 s}$, THEN

$$
\begin{aligned}
\frac{\partial u}{\partial t}= & \nabla \cdot\left(\mathcal{D}(t, x, u) \circ \nabla_{p} u\right)-\mathscr{B}(u)+\mathcal{C}_{i 2}(t) f(u) \\
& +\mathcal{D}_{i 2}(t) g\left(u\left(t-\tau_{i}(t), x\right)\right), \quad i \in S,
\end{aligned}
$$

where $\Omega=\left\{\left(x_{1}, x_{2}\right)^{T} \in R^{2}:\left|x_{k}\right|<\sqrt{2}, k=1,2\right\}, x=\left(x_{1}, x_{2}\right)^{T} \in \Omega, u=\left(u_{1}^{T}(t, x), u_{2}^{T}(t, x)\right)^{T} \in$ $R^{2}$, and we select the Neumann boundary condition. Let $S=\{1,2\}$ and $\pi_{11}=-0.8, \pi_{12}=0.8$; $\pi_{21}=0.5, \pi_{22}=-0.5$.

$$
\begin{aligned}
& \mathcal{C}_{11}=\left(\begin{array}{cc}
0.2 & 0.01 \\
0.01 & 0.1
\end{array}\right), \quad \mathcal{C}_{12}=\left(\begin{array}{cc}
0.1 & 0.01 \\
0.01 & 0.2
\end{array}\right), \quad \mathcal{C}_{21}=\left(\begin{array}{cc}
0.3 & 0.01 \\
0.01 & 0.1
\end{array}\right), \\
& \mathcal{C}_{22}=\left(\begin{array}{cc}
0.1 & 0.01 \\
0.01 & 0.3
\end{array}\right), \quad \mathcal{D}_{11}=\left(\begin{array}{cc}
0.2 & -0.02 \\
-0.02 & 0.1
\end{array}\right), \quad \mathcal{D}_{12}=\left(\begin{array}{cc}
0.1 & -0.02 \\
-0.02 & 0.2
\end{array}\right), \\
& \mathcal{D}_{21}=\left(\begin{array}{cc}
0.3 & -0.02 \\
-0.02 & 0.1
\end{array}\right), \quad \mathcal{D}_{22}=\left(\begin{array}{cc}
0.1 & -0.02 \\
-0.02 & 0.3
\end{array}\right), \\
& E_{11}=E_{12}=E_{21}=E_{22}=\left(\begin{array}{cc}
0.01 & 0.001 \\
0.001 & 0.02
\end{array}\right) ;
\end{aligned}
$$




$$
\begin{aligned}
& H_{11}=H_{12}=H_{21}=H_{22}=\left(\begin{array}{cc}
0.02 & 0.001 \\
0.001 & 0.01
\end{array}\right)=M_{11}=M_{12}=M_{21}=M_{22} ; \\
& \mathbb{B}=\left(\begin{array}{cc}
1.4 & 0 \\
0 & 1.5
\end{array}\right), \quad F_{2}=\left(\begin{array}{ll}
1 & 0 \\
0 & 1
\end{array}\right)=G_{2}, \quad F_{1}=\left(\begin{array}{ll}
0 & 0 \\
0 & 0
\end{array}\right)=G_{1} .
\end{aligned}
$$

Let $\beta=0.001$ and $\tau(t) \equiv \tau=126.8$. According to Theorem 3.1, we can solve LMI conditions (3.2) by using Matlab LMI Toolbox and obtain $t$ min $=-0.0017<0$, which implies feasible (see [3, Remark 29(3)] for detail). Further, the corresponding matrices are extracted as follows:

$$
\begin{aligned}
& P_{1}=\left(\begin{array}{cc}
14.8733 & 0 \\
0 & 14.7277
\end{array}\right), \quad P_{2}=\left(\begin{array}{cc}
12.8235 & 0 \\
0 & 12.7377
\end{array}\right) \text {, } \\
& Q=\left(\begin{array}{cc}
5.4959 & 0 \\
0 & 6.3540
\end{array}\right), \quad L_{1}=\left(\begin{array}{cc}
5.0231 & 0 \\
0 & 5.4188
\end{array}\right) \text {, } \\
& L_{2}=\left(\begin{array}{cc}
4.8404 & 0 \\
0 & 5.5999
\end{array}\right) \text {. }
\end{aligned}
$$

Hence, we conclude from Theorem 3.1 that PDEs (4.1)-(4.2) is global exponential robust stability in the mean square.

Remark 4.1 Example 4.1 illustrates the effectiveness and less conservatism due to the allowable upper bound of time-delay $(\tau=126.8)$.

\section{Conclusions and further studies}

The stability of the nonlinear $p$-Laplace $(p>1)$ Markovian jumping dynamic PDEs was first studied in [17]. Since then, there have been a lot of related literature [1-3, 16-18] involving the stability analysis of the nonlinear $p$-Laplace $(p>1)$ dynamic PDEs under various complicated and practical factors, such as impulse, parameter uncertainties and so on. However, in all the previous related literature, the existence of solutions of those PDEs was neglected. Naturally, people want to know whether there are other solutions besides the trivial solution. If there exists only the trivial solution as the unique solution for the PDEs, all those stability criteria about the trivial solution would become meaningless though these PDEs of all the previous related literature can actually own infinitely many solutions only if the similar sufficient conditions are also given. So, in this paper, we present a sufficient condition for the existence of PDEs (1.6) in our Theorem 3.1 by way of the contraction mapping theory. Moreover, we have provided the methods, by which the existence of solutions for those PDEs in the above related literature can similarly be proved. The works of all the above related literature become more meaningful owing to the contribution of this paper (see Remark 3.2). So the further study is no longer the existence of solutions for dynamic PDEs with the nonlinear $p$-Laplace.

Note that almost all the above related literature did not point out the role that the nonlinear $p$-Laplace items play, except [17]. In fact, when $p=2$, 2-Laplace is the linear Laplace, and there are many papers (see, e.g., $[9,10,19-21])$ in which the Laplace diffusion item plays its role in their stability criteria for the linear Laplace PDEs can be considered in the special Hilbert space $H^{1}(\Omega)$ that can be orthogonally decomposed into the direct sum 
of infinitely many eigenfunction spaces. However, the nonlinear $p$-Laplace $(p>1, p \neq 2)$ brings great difficulties, for the nonlinear $p$-Laplace PDEs should be considered in the frame of Sobolev space $W^{1, p}(\Omega)$ that is only a reflexive Banach space. Indeed, owing to the great difficulties, the authors only provide in [17] the stability criterion in which the nonlinear $p$-Laplace items play roles in the case of $1<p<2$. So a further profound study is very interesting, which may call for some new mathematical methods, and even new mathematical theories. Under the Dirichlet or Neumann boundary condition, the problem of the role of the nonlinear $p$-Laplace $(p>2$ or $p>1)$ item in the stability criteria for PDEs still remains open and challenging.

\section{Competing interests}

The authors declare that they have no competing interests.

\section{Authors' contributions}

All authors typed, read and approved the final manuscript. ZP is the first author and the corresponding author, and RR is the second author.

\section{Acknowledgements}

The authors would like to thank the anonymous referees for their detailed comments and valuable suggestions which considerably improved the presentation of this paper. This research is supported by the Scientific Research Fund of Science Technology Department of Sichuan Province (2010JY0057, 2012JYZ010), and by the Scientific Research Fund of Sichuan Provincial Education Department (12ZB349).

Received: 19 September 2013 Accepted: 8 November 2013 Published: 03 Dec 2013

\section{References}

1. Wang, X, Rao, R, Zhong, S: LMI approach to stability analysis of Cohen-Grossberg neural networks with $p$-Laplace diffusion. J. Appl. Math. 2012, Article ID 523812 (2012)

2. Pan, Q, Zhang, Z, Huang, J: Stability of the stochastic reaction-diffusion neural network with time-varying delays and p-Laplacian. J. Appl. Math. 2012, Article ID 405939 (2012)

3. Rao, R, Wang, X, Zhong, S, Pu, Z: LMI approach to exponential stability and almost sure exponential stability for stochastic fuzzy Markovian-jumping Cohen-Grossberg neural networks with nonlinear p-Laplace diffusion. J. Appl. Math. 2013, Article ID 396903 (2013)

4. Wang, Y, Xie, L, Souza, D, Carlos, E: Robust control of a class of uncertain nonlinear system. Syst. Control Lett. 19(2), 139-149 (1992)

5. Boyd, S, Ghaoui, L, Feron, F, Balakrishnan, V: Linear Matrix Inequalities in Systems and Control Theory. SIAM, Philadelphia (1994)

6. Zhu, Q, Li, X, Yang, X: Exponential stability for stochastic reaction-diffusion BAM neural networks with time-varying and distributed delays. Appl. Math. Comput. 217(13), 6078-6091 (2011)

7. Sathy, R, Balasubramaniam, P: Stability analysis of fuzzy Markovian jumping Cohen-Grossberg BAM neural networks with mixed time-varying delays. Commun. Nonlinear Sci. Numer. Simul. 16(4), 2054-2064 (2011)

8. Zhu, Q, Li, X: Exponential and almost sure exponential stability of stochastic fuzzy delayed Cohen-Grossberg neural networks. Fuzzy Sets Syst. 203, 74-94 (2012)

9. Zhou, C, Zhang, H, Zhang, H, Dang, C: Global exponential stability of impulsive fuzzy Cohen-Grossberg neural networks with mixed delays and reaction-diffusion terms. Neurocomputing 91, 67-76 (2012)

10. Wang, C, Kao, Y, Yang, G: Exponential stability of impulsive stochastic fuzzy reaction-diffusion Cohen-Grossberg neural networks with mixed delays. Neurocomputing 89, 55-63 (2012)

11. Song, Q, Cao, J: Global dissipativity on uncertain discrete-time neural networks with time-varying delays. Discrete Dyn. Nat. Soc. 2010, Article ID 810408 (2010)

12. Song, Q, Wang, Z, Liang, J: Analysis on passivity and passification of T-S fuzzy systems with time-varying delays. J. Intell. Fuzzy Syst. 24(1), 21-30 (2013)

13. Song, Q: Stochastic dissipativity analysis on discrete-time neural networks with time-varying delays. Neurocomputing 74(5), 838-845 (2011)

14. Song, Q, Wang, Z: New results on passivity analysis of uncertain neural networks with time-varying delays. Int. J. Comput. Math. 87(3), 668-678 (2010)

15. $\mathrm{Xu}, \mathrm{D}, \mathrm{Xu}, \mathrm{L}$ : New results for studying a certain class of nonlinear delay differential systems. IEEE Trans. Autom. Control 55(7), 1641-1645 (2010)

16. Rao, R, Zhong, S, Wang, X: Delay-dependent exponential stability for Markovian jumping stochastic Cohen-Grossberg neural networks with $p$-Laplace diffusion and partially known transition rates via a differential inequality. Adv. Differ. Equ. 2013, 183 (2013)

17. Rao, R, Zhong, S, Wang, X: Stochastic stability criteria with LMI conditions for Markovian jumping impulsive BAM neural networks with mode-dependent time-varying delays and nonlinear reaction-diffusion. Commun. Nonlinear Sci. Numer. Simul. 19(1), 258-273 (2014)

18. Rao, R, Pu, Z: Stability analysis for impulsive stochastic fuzzy $p$-Laplace dynamic equations under Neumann or Dirichlet boundary condition. Bound. Value Probl. 2013, 133 (2013) 
19. Pan, J, Zhong, S: Dynamical behaviors of impulsive reaction-diffusion Cohen-Grossberg neural network with delays. Neurocomputing 73, 1344-1351 (2010)

20. Pan, J, Liu, X, Zhong, S: Stability criteria for impulsive reaction-diffusion Cohen-Grossberg neural networks with time-varying delays. Math. Comput. Model. 51, 1037-1050 (2010)

21. Pan, J, Zhong, S: Dynamic analysis of stochastic reaction-diffusion Cohen-Grossberg neural networks with delays. Adv. Differ. Equ. 2009, Article ID 410823 (2009)

22. Xu, D, Wang, X, Yang, Z: Further results on existence-uniqueness for stochastic functional differential equations. Sci. China Math. 56(6), 1169-1180 (2013)

23. Li, B, Li, D, Xu, D: Stability analysis for impulsive stochastic delay differential equations with Markovian switching. J. Franklin Inst. 350(7), 1848-1864 (2013)

24. Rao, R, Pu, Z: LMI-based stability criterion of impulsive T-S fuzzy dynamic equations via fixed point theory. Abstr. Appl. Anal. 2013, Article ID 261353 (2013)

10.1186/1687-2770-2013-264

Cite this article as: Pu and Rao: Exponential robust stability of T-S fuzzy stochastic $p$-Laplace PDEs under zero-boundary condition. Boundary Value Problems 2013, 2013:264

\section{Submit your manuscript to a SpringerOpen ${ }^{\circ}$ journal and benefit from:}

- Convenient online submission

- Rigorous peer review

- Immediate publication on acceptance

Open access: articles freely available online

- High visibility within the field

- Retaining the copyright to your article 